\title{
Application of the Triangular Fuzzy Model to Assessment of Analogical Reasoning Skills
}

\author{
Michael Gr. Voskoglou ${ }^{1, *}$, Igor Ya. Subbotin ${ }^{2}$ \\ ${ }^{1}$ School of Technological Applications, Graduate Technological Educational Institute (T. E. I.) of Western Greece, Patras, Greece \\ ${ }^{2}$ College of Letters and Sciences, National University of Los Angeles, California, USA \\ *Corresponding author: mvosk@hol.gr
}

Received December 20, 2014; Revised January 04, 2015; Accepted January 06, 2015

\begin{abstract}
We apply the Triangular Fuzzy Assessment Model (TFAM) for analogical reasoning skills assessment. The TFAM is a new original model based on the Centre of Gravity (COG) defuzzification technique, which we have properly adapted and used in earlier papers as an assessment method of several human activities. The main idea of the TFAM is the replacement of the rectangles appearing in the graph of the COG technique by isosceles triangles sharing common parts. In this way, we can treat better the ambiguous cases of scores being at the boundaries between two successive linguistic characterizations (grades) of the level of the individuals' performance. An application is also presented illustrating our results in practice, where the TFAM is compared with two traditional assessment methods (calculation of the means and of the GPA index) based on principles of the bivalent logic (yesno).
\end{abstract}

Keywords: Analogical Reasoning, Fuzzy Logic, Centre of Gravity (COG) defuzzification technique, Triangular Fuzzy Assessment model (TFAM), GPA index

Cite This Article: Michael Gr. Voskoglou, and Igor Ya. Subbotin, "Application of the Triangular Fuzzy Model to Assessment of Analogical Reasoning Skills." American Journal of Applied Mathematics and Statistics, vol. 3, no. 1 (2015): 1-6. doi: 10.12691/ajams-3-1-1.

\section{Introduction}

In 1999 Voskoglou developed a fuzzy model for the description of the learning process [15,18] and later [17] he used the total uncertainty of the corresponding fuzzy system for assessing the students' skills in learning mathematics. Meanwhile Subbotin et al. [9], based on Voskoglou's [15] fuzzy model, adopted properly the widely used in Fuzzy Mathematics Center of Gravity (COG) defuzzification technique to provide an alternative measure for the assessment of students' learning skills. Since then, both Voskoglou and Subbotin, either collaborating or independently to each other, utilized the COG technique in assessing other students' competencies (e.g. see [11,14,20,21], etc) as well as the Bridge player's performance [22] and for testing the effectiveness of a CBR system [10].

The first attempt to apply the Triangular Fuzzy Assessment Model (TFAM) was made in [12], while more recently Subbotin and Voskoglou [13] presented an improved version of it for assessing students' critical thinking skills. The main idea of the TFAM is to replace the rectangles appearing in the membership function's graph of our COG assessment model by triangles sharing common parts, which must be considered twice in calculating the COG of the level's area lying between the resulting graph and the OX axis. In this way, one succeeds to treat better the ambiguous cases of students' scores being at the boundaries between two successive grades. It is a very common approach in such cases to divide the interval of the specific grades in three parts and to assign the corresponding grade using + and -. For example, in a scale of scores from 0 to 100 we could have $75-77=\mathrm{B}$-, $78-80=\mathrm{B}, 81-84=\mathrm{B}+$. However, this consideration does not reflect the common situation, where the teacher is not sure about the grading of the students whose performance could be assessed as marginal between and close to two adjacent grades; for example, something like between 74 (C) and 75 (B). The TFAM fits to this kind of situations.

This paper aims at using an extended version of the TFAM for obtaining a fuzzy measure of students' Analogical Reasoning (AR) skills. The rest of the paper is organized as follows: In section 2 we describe the AR process. In section 3 we develop the TFAM using a scale of five grades (A, B, C, D and F) instead of the three grades (A, B, C-F) used in [12] and [13]. In this way the assessment method obtained becomes more accurate. In section 4 we present a classroom application performed recently with students of the Graduate Technological Educational Institute (T. E. I.) of Western Greece illustrating our results in practice. Finally, section 5 is devoted to conclusions and discussion about our plans for further research on the subject.

For general facts on fuzzy sets we refer to the book [7].

\section{Analogical Reasoning}


Reasoning is the most important human brain operation. In particular, much of our cognitive activity does depend on our ability to reason analogically. Analogies are used for explaining concepts which cannot directly perceived (e.g. electricity in terms of the water flow), in making predictions within domains, in communication and persuasion, etc.

AR is actually a method of processing information that compares the similarities between new and past understood concepts, then using these similarities to gain understanding of the new concept.

AR is important in general for creativity and scientific discovery. Within cognitive science mental processes are likened to computer programs (e.g. neural networks) and such analogies serve as mental models to support reasoning in new domains. In Artificial Intelligence the Case-Based-Reasoning paradigm covers a range of different methods (including AR) for organizing, retrieving, utilizing and indexing the knowledge of past cases [19,23].

Solution of problems by analogy (analogical problem solving) is probably the most important aspect of AR. However this strategy can be difficult to implement in problem solving, because it requires the solver to attend to information other than the problem to be solved (usually referred as the target problem). Thus the solver may come up empty-handed, either because he/she has not solved any similar problems in past, or because he/she fails to realize the relevance of previous problems. But, even if an analogue is retrieved, the solver must know how to use it to determine the solution procedure for the target problem.

A characteristic example is the experiment of Gick and Holyoak [3,4] on a problem known as the Dunker's tumor problem: You have a patient with an inoperable stomach tumor. There are some rays that, at sufficient intensity, destroy organic tissue. How can you free the patient of the tumor without destroying the healthy tissue surrounding it?

The desired solution is to use a system of multiple machines to emit low-intensity rays from different directions. These rays will converge on the tumor and their combined effect will destroy it.

In the first phase of the experiment only a $10 \%$ of the subjects gave the correct solution. Next, before presenting the problem to another group of subjects, an analogous story was given to read about a general, who wants to capture a fortress and he is able to do so by sending parts of his army down each of several roads, all of which converge on the fortress. In this case the percentage of the correct solutions increased to the $30 \%$ of the subjects, while a further spectacular increase to $70 \%$ happened when subjects were given the hint to use the story above for the solution of the target problem.

Several studies (e.g. [1,2,6,8,16,23], etc) have provided detailed models for the process of AR, which are broadly consistent with reviews of problem solving strategy training studies, in which factors associated with instances of successful transfer - that is, use of already existing knowledge to produce new knowledge - are identified. According to these studies the main steps of the AR process include:

- Representation of the target problem.

- Search-retrieval of a related past problem.

- Mapping of the representations of the target and the related problem.
- Adaptation of the solution of the related problem for use with the target problem.

More specifically, before solvers working on a problem they usually construct a representation of it. A good representation must include both the surface and the structural (abstract, solution relevant) features of the problem. The former are mainly determined by what are the quantities involved in the problem and the latter by how these quantities are related to each other. The features included in solvers' representations of the target problem are used as retrieval cues for a related problem in memory, usually referred as a source problem. When the two problems share structural but not surface features the source is called a remote analogue of the target problem.

Analogical mapping requires aligning the two situations - that is, finding the correspondences between the representations of the target and the source problem - and projecting inferences from the source to the target problem. Once the common alignment and the candidate inferences have been discovered, the analogy is evaluated. The last step involves the adaptation of the solution of the analogous problem for use with the target problem, where the correspondences between objects and relations of the two problems must be used.

The successful completion of the AR process is referred as positive analogical transfer. But the search may also yield distracting problems having surface but not structural (solution relevant) common features with the target problem and therefore being only superficially similar to it. The reason for this is usually a non satisfactory representation of the target problem, containing only its salient surface features, and the resulting consequences on the retrieval cues available for the search process. When a distracting problem is considered as an analogue of the target, we speak about negative analogical transfer. This happens when a distracting problem is retrieved as a source problem and the solver fails, through the mapping of the representations of the source and target problem, to realize that the source cannot be considered as an analogue to the target. Therefore the process of mapping is very important in AR playing the role of a "control system" for the fitness of the source problem.

\section{The Triangular Fuzzy Assessment Model}

Let $\mathrm{U}=\{\mathrm{A}, \mathrm{B}, \mathrm{C}, \mathrm{D}, \mathrm{F}\}$ be the set of students' grades $\mathrm{A}=$ excellent, $\mathrm{B}=$ very good, $\mathrm{C}=\operatorname{good}, \mathrm{D}=$ fair and $\mathrm{F}=$ not satisfactory. In applying the COG as an assessment method, we represent the student group under assessment as a fuzzy set in $U$ and we associate to each $x$ in $U$ an interval of the $\mathrm{OX}$ axis (i.e. we replace $U$ with a set of real intervals). Then, we construct the graph of the corresponding membership function, which, in this particular case, is a bar graph consisting of five rectangles with one side lying on the $\mathrm{OX}$ axis (e.g. see Figure 1 of [22]). Next, using the well known from Mechanics formulas, we calculate the coordinates $\left(X_{c}, Y_{c}\right)$ of the COG of the level's section lying between this graph and the $\mathrm{OX}$ axis, thus obtaining the defuzzification of the corresponding fuzzy data. Further, using elementary algebraic inequalities we determine the area where the 
COG lies and by elementary geometric observations we obtain a criterion about the student group's performance (for details see, for example, section 2 of [22]).

As said in our introduction, for applying the TFAM instead of the classical COG technique, we replace the rectangles appearing in the graph of the COG technique by triangles. Therefore, we shall have five such triangles in the resulting scheme, each one corresponding to a students' grade (F, D, C, B and A respectively). Without loss of generality and for making our calculations easier we consider isosceles triangles with bases of length 10 units lying on the OX axis. The height to the base of each triangle is equal to the percentage of students' of the group under assessment who achieved the corresponding grade. We allow for any two adjacent triangles to have $30 \%$ of their bases belonging to both of them. In this way, we cover the situation of uncertainty in assessing marginal students' scores, as we have described in our introduction.

The resulting scheme is presented in Figure 1. The student group under assessment can be represented again, as in the COG technique, as a fuzzy set in U, whose membership function $y=m(x)$ has as graph the line $\mathrm{OA}_{1} \mathrm{~B}_{1} \mathrm{~A}_{2} \mathrm{~B}_{2} \mathrm{~A}_{3} \mathrm{~B}_{3} \mathrm{~A}_{4} \mathrm{~B}_{4} \mathrm{~A}_{5} \mathrm{C}_{9}$. It is easy then to calculate the coordinates $\left(b_{i 1}, b_{i 2}\right)$ of the points $B_{i}, i=1,2,3,4$, 5. In fact, $B_{1}$ is the intersection of the straight line segments $A_{1} C_{2}$ and $C_{1} A_{2}, B_{2}$ is the intersection of $C_{3} A_{3}$, and $A_{2} C_{4}$ and so on. Therefore, it is straightforward to determine the analytic form of $y=m(x)$ consisting of 10 branches, corresponding to the equations of the straight lines $\mathrm{OA}_{1}$, $A_{1} B_{1}, B_{1} A_{2}, A_{2} B_{2}, B_{2} A_{3}, A_{3} B_{3}, B_{3} A_{4}, A_{4} B_{4}, B_{4} A_{5}$ and $\mathrm{A}_{5} \mathrm{C}_{9}$ in the intervals $[0,5),\left[5, b_{11}\right),\left[b_{11}, 12\right),\left[12, b_{12}\right),\left[b_{12}\right.$, 19), $\left[19, b_{13}\right),\left[b_{13}, 26\right),\left[26 . b_{14}\right),\left[b_{14}, 33\right)$ and $[33,38]$ respectively.

However, when applying the TFAM, the use of the analytic form of $y=m(x)$ is not needed (in contrast to the case of the classical COG technique) for the calculation of the COG of the resulting level's area. In fact, since the marginal cases of students' grades should be considered as common parts for any pair of the adjacent triangles, it is logical to don't subtract the areas of the intersections from the area of the corresponding level's section, although in this way we count them twice; e.g. placing the ambiguous cases $\mathrm{B}+$ and $\mathrm{A}-$ in both regions $\mathrm{B}$ and $\mathrm{A}$. In other words, the classical COG technique, which calculates the coordinates of the COG of the area between the graph of the membership function (line $\mathrm{OA}_{1} \mathrm{~B}_{1} \mathrm{~A}_{2} \mathrm{~B}_{2} \mathrm{~A}_{3} \quad \mathrm{~B}_{3} \mathrm{~A}_{4}$ $\mathrm{B}_{4} \mathrm{~A}_{5} \mathrm{C}_{9}$ ) and the $\mathrm{OX}$ axis (see Figure 1 ), thus considering the areas of the "common" triangles $\mathrm{C}_{1} \mathrm{~B}_{1} \mathrm{C}_{2}, \mathrm{C}_{3} \mathrm{~B}_{2} \mathrm{C}_{4}$, $\mathrm{C}_{5} \mathrm{~B}_{3} \mathrm{C}_{6}$ and $\mathrm{C}_{7} \mathrm{~B}_{4} \mathrm{C}_{8}$ only once, is not the proper one to be applied in the above situation.

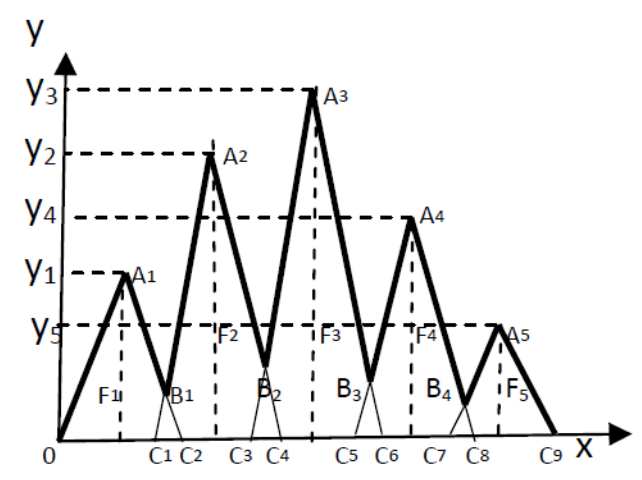

Figure 1. The membership function's graph of TFAM
Indeed, in this case it is reasonable to represent each one of the five triangles $\mathrm{OA}_{1} \mathrm{C}_{2}, \mathrm{C}_{1} \mathrm{~A}_{2} \mathrm{C}_{4}, \mathrm{C}_{3} \mathrm{~A}_{3} \mathrm{C}_{6}, \mathrm{C}_{5} \mathrm{~A}_{4} \mathrm{C}_{8}$ and $\mathrm{C}_{7} \mathrm{~A}_{5} \mathrm{C}_{9}$ of Figure 1 by their COG's $\mathrm{F}_{\mathrm{i}}, \mathrm{i}=1,2,3,4,5$ and to consider the entire level's area as the system of these points-centers. More explicitly, the steps of the whole construction of the TFAM are the following:

1. Let $y_{1}, y_{2}, y_{3}, y_{4}, y_{5}$ be the percentages of the students in the group getting $\mathrm{F}, \mathrm{D}, \mathrm{C}, \mathrm{B}$, and A grades respectively, then $y_{1}+y_{2}+y_{3}+y_{4}+y_{5}=1(100 \%)$.

2. We consider the isosceles triangles with bases having lengths of 10 units each and their heights $y_{1}, y_{2}, y_{3}, y_{4}, y_{5}$ in the way that has been illustrated in Figure 1. Each pair of adjacent triangles has common parts in the base with length 3 units.

3. We calculate the coordinates $\left(x_{c_{i}}, y_{c_{i}}\right)$ of the COG $F_{i}, i=1,2,3,4,5$ of each triangle as follows: The COG of a triangle is the point of intersection of its medians, and since this point divides the median in proportion 2:1 from the vertex, we find, taking also into account that the triangles are isosceles, that $y_{c_{i}}=\frac{1}{3} y_{i}$. Further, since the triangles' bases have a length of 10 units, it is easy to observe that $x_{c i}=7 i-2$.

4. We consider the system of the centers $F_{i}, i=1,2,3,4$, 5 and we calculate the coordinates $\left(\mathrm{X}_{\mathrm{c}}, \mathrm{Y}_{\mathrm{c}}\right)$ of the COG $\mathrm{F}_{\mathrm{C}}$ of the whole level's area considered in Figure 1 from the following formulas, derived from the commonly used in such cases definition (e.g. see [24]):

$$
X_{C}=\frac{1}{S} \sum_{i=1}^{5} S_{i} x_{c_{i}}, Y_{C}=\frac{1}{S} \sum_{i=1}^{5} S_{i} y_{c_{i}}
$$

In formulas (1) $S$ denotes the whole of the considered level's area and $S i, \mathrm{i}=1,2,3,4,5$ denote the areas of the corresponding triangles. Therefore $\mathrm{Si}=5 \mathrm{y}_{\mathrm{i}}$ and $S=\sum_{i=1}^{5} S_{i}=5 \sum_{i=1}^{5} y_{i}=5$. Thus, from formulas (1) we finally get:

$$
\begin{gathered}
X_{C}=\frac{1}{5} \sum_{i=1}^{5} 5 y_{i}(7 i-2)=7 \sum_{i=1}^{5} i y_{i}-2 \\
Y_{C}=\frac{1}{5} \sum_{i=1}^{5} 5 y_{i}\left(\frac{1}{3} y_{i}\right)=\frac{1}{5} \sum_{i=1}^{5} y_{i}^{2}
\end{gathered}
$$

5. We determine the area where the COG $F_{c}$ lies as follows: For i, $j=1,2,3,4,5$, we have that

$0 \leq\left(y_{i}-y_{j}\right)^{2}=y_{i}^{2}+y_{j}^{2}-2 y_{i} y_{j}$, therefore $y_{i}^{2}+y_{j}^{2} \geq 2 y_{i} y_{j}$, with the equality holding if, and only if, $\mathrm{y}_{\mathrm{i}}=\mathrm{y}_{\mathrm{j}}$. Therefore

$$
\begin{aligned}
& 1=\left(\sum_{i=1}^{5} y_{i}\right)^{2}=\sum_{i=1}^{5} y_{i}^{2}+2 \sum_{\substack{i, j=1, i \neq j}}^{5} y_{i} y_{j} \\
& \leq \sum_{i=1}^{5} y_{i}^{2}+\sum_{\substack{i, j=1, i \neq j}}^{5}\left(y_{i}^{2}+y_{j}^{2}\right)=5 \sum_{i=1}^{5} y_{i}^{2}, \text { or } \sum_{i=1}^{5} y_{i}^{2} \geq \frac{1}{5}
\end{aligned}
$$

with the equality holding if, and only if, $\mathrm{y}_{1}=\mathrm{y}_{2}=\mathrm{y}_{3}=\mathrm{y}_{4}=$ $\mathrm{y}_{5}=\frac{1}{5}$. In the case of equality the first of formulas gives that $X_{c}=7\left(\frac{1}{5}+\frac{2}{5}+\frac{3}{5}+\frac{4}{5}+\frac{5}{5}\right)-2=19$. Further, 
combining the inequality (3) with the second of formulas (2) one finds that $Y_{c} \geq \frac{1}{25}$ Therefore the unique minimum for $Y_{c}$ corresponds to the COG $F_{m}\left(19, \frac{1}{25}\right)$. The ideal case is when $y_{1}=y_{2}=y_{3}=y_{4}=0$ and $y_{5}=1$. Then from formulas (2) we get that $X_{c}=33$ and $Y_{c}=\frac{1}{5}$.Therefore the COG in this case is the point $F_{i}\left(33, \frac{1}{5}\right)$. On the other hand, the worst case is when $y_{1}=1$ and $y_{2}=y_{3}=y_{4}=y_{5}=0$. Then from formulas (2), we find that the COG is the point $F_{w}\left(5, \frac{1}{5}\right)$. Therefore the "area" where the COG $F_{c}$ lies is the triangle $F_{w} F_{m} F_{i}$ presented in Figure 2.

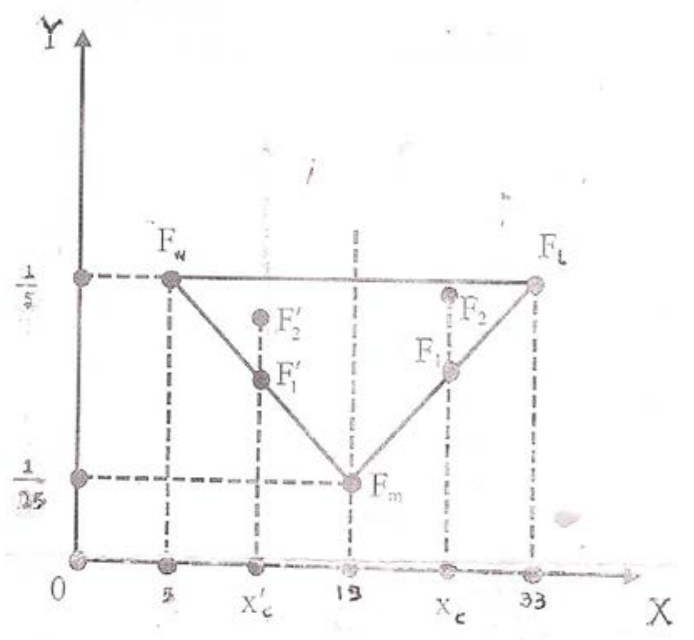

Figure 2. The area where the COG lies

6. We formulate our criterion for comparing the performance of two (or more) groups' as follows: From elementary geometric observations (see Figure 2) it follows that for two groups the group having the greater $X_{c}$ performs better. Further, if the two groups have the same $X_{c} \geq 19$, then the group having the COG which is situated closer to $F i$ is the group with the greater $Y_{c}$. Also, if the two groups have the same $X_{c}<19$, then the group having the COG which is situated farther to $F w$ is the group with the smaller $Y_{c}$. Based on the above considerations it is logical to formulate our criterion for comparing the two groups' performance in the following form:

- Among two or more groups the group with the greater $X_{c}$ performs better.

- If two or more groups have the same $X_{c} \geq 19$, then the group with the greater $Y_{c}$ performs better.

- If two or more groups have the same $X_{c}<19$, then the group with the lower $Y_{c}$ performs better.

\section{An Application to AR}

In order to illustrate the use of the TFAM as an assessment method of AR skills we performed the following experiment, in which the subjects were students of the Graduate Technological Educational Institute of Western Greece, in the city of Patras. We formed two groups, say $G_{1}$ and $G_{2}$, of 34 and 51 students respectively from two different Departments of the School of Technological Applications (prospective engineers) being at their first term of studies.

Two mathematical problems were given for solution to both groups (see Appendix). The first of them was on combinatorial analysis and probability and the second was on the theory of matrices, which are topics of the students' first term course of mathematics. In each case and before receiving the target problem students received three other problems together with their solution procedures. They read each problem and its solution procedure and then solved in detail the problem themselves using the given procedure. Students were allowed 10 minutes for each problem and they were not given the other problem until after 10 minutes had elapsed. The first of these problems was a remote analogue of the target problem, the second was a distracting problem, while the third was unrelated to the target problem. Next the target problem was given and it was asked to students to try to solve it by adapting the solution of one of the previous three problems (time allowed 20 minutes).

The students' solutions of the target problems were marked in a scale from 0 to 100 and their performance was characterized as follows: A (excellent) $=85-100$, B $($ very good $)=75-84, \mathrm{C}($ good $)=60-74, \mathrm{D}($ fair $)=50-59$ and $\mathrm{F}$ (not satisfactory) $=0-49$

The scores achieved by the students of the two groups were the following:

First group $\left(\mathrm{G}_{1}\right)$ : 100(1 time), 98(1), 95(3), 94(2), 93(1), 88(1), 85(3), 82(4), 80(3), 76(1), 74(2), 73(1), 63(1), 59(2), 58(1), 55(2), 52(1), 48(1), 45(2), 38(1).

Second group $\left(\mathrm{G}_{2}\right)$ : 99(1), 95(3), 88(3), 85(5), 82(3), 80(2), 78(4), 75(9), 70(2), 64(3), 60(4), 58(2), 56(3), 50(4), 45(1), 40(1), 26(1).

The above data is summarized in the following Table:

Table 1. Students' grades
\begin{tabular}{|c|c|c|}
\hline Grades & $\mathrm{G}_{1}$ & $\mathrm{G}_{2}$ \\
\hline A & 12 & 12 \\
\hline B & 8 & 18 \\
\hline C & 4 & 9 \\
\hline D & 6 & 9 \\
\hline F & 4 & 3 \\
\hline Total & 34 & 51 \\
\hline
\end{tabular}

The evaluation of this data will be performed below in two ways: i) By using two traditional assessment methods based on principles of the bivalent logic (yes-no) and ii) by applying the TFAM. The results obtained will be discussed and compared to each other.

\subsection{Traditional Methods}

a) Calculation of the means: Adding all students' scores (presented above) for each group and dividing the corresponding sum by the total number of students of the group (34 for $G_{1}$ and 51 for $G_{2}$ ) one finds that the means of the students' scores are approximately 74.97 and 71.24 for the first and the second group respectively. This shows that the mean performance of both groups was good, with the performance of the first group being better.

b) Calculation of the GPA index: We recall that the Great Point Average (GPA) index is a weighted mean, where more importance is given to the higher scores, to which greater coefficients (weights) are attached (e.g. see 
[5]). In other words, the GPA index focuses on the quality performance of a student group.

In fact, let us denote by $n_{A}, n_{B}, n_{C}, n_{D}$ and $n_{E}$ the numbers of students whose performance was characterized by $\mathrm{A}, \mathrm{B}, \mathrm{C}, \mathrm{D}$ and $\mathrm{F}$ respectively and by $n$ the total number of students of each group.. Then the GPA index is calculated by the formula $\mathrm{GPA}=\frac{n_{D}+2 n_{C}+3 n_{B}+4 n_{A}}{n}$. In terms of the notation used in section 3 the above formula can be written in the form GPA $=y_{2}+2 y_{3}+3 y_{4}+4 y_{5}(4)$.

In the ideal case $\left(\mathrm{y}_{1}=\mathrm{y}_{2}=\mathrm{y}_{3}=\mathrm{y}_{4}=0, \mathrm{y}_{5}=1\right)$ the equation (4) gives that GPA $=4$, while in the worst case $\left(\mathrm{y}_{1}=1, \mathrm{y}_{2}=\mathrm{y}_{3}=\mathrm{y}_{4}=\mathrm{y}_{5}=0\right)$ it gives that GPA $=0$. Therefore we have that $0 \leq \mathrm{GPA} \leq 4$.

In our case, from Table 1 one finds that the percentages of the students in each group getting the grades $\mathrm{F}, \mathrm{D}, \mathrm{C}, \mathrm{B}$, and $\mathrm{A}$ respectively are $y_{1}=\frac{4}{34}, y_{2}=\frac{6}{34}, y_{3}=\frac{4}{34}, y_{4}=\frac{8}{34}, y_{5}=\frac{12}{34}$ for $G_{1}$ and $y_{1}=\frac{3}{51}, y_{2}=\frac{9}{51}, y_{3}=\frac{9}{51}, y_{4}=\frac{18}{51}, y_{5}=\frac{12}{51}$ for $\mathrm{G}_{2}$. Therefore (4) gives that $\mathrm{GPA}=\frac{6}{34}+2 \cdot \frac{4}{34}+3 \cdot \frac{8}{34}+4 \cdot \frac{12}{34}=\frac{43}{17} \approx 2.529$ for $\mathrm{G}_{1}$. Similarly one finds that the GPA index takes the same value for $G_{2}$. Thus, since the above value of the GPA index is greater than the half of its maximal value, which is equal to 4, the two student groups demonstrated a satisfactory quality performance.

\subsection{Application of the TFAM}

From the description of the TFAM presented in section 3 it becomes evident that in this case a group's performance is actually measured by the abscissa $X_{c}$ of the COG. Thus, observing the first of formulas (2) it is easy to realize that the TFAM, similarly with the GPA index, measures the group's quality performance.

In the case of our experiment, applying the first of formulas (2) on the data of Table 1 it is straightforward to check that the value of $X_{c}$ for both groups is equal to $\frac{386}{17} \approx 22.71>19$. In fact, according to the students' percentages (see calculation of the GPA index in section 4.1) we find for $\mathrm{G}_{1}$ that $X_{c}=$ $7\left(\frac{4}{34}+2 \cdot \frac{6}{34}+3 \cdot \frac{4}{34}+4 \cdot \frac{8}{34}+5 \cdot \frac{12}{34}\right)-2=\frac{772}{34}$ and a similar argument holds for $G_{2}$. Thus, since the value 22.71 is much greater than the half of the value 33 of $X_{c}$ in the ideal case, both groups demonstrated a good quality performance.

Further, from the second of formulas (2) one finds that $Y_{c}=\frac{1}{5} \cdot\left[\left(\frac{4}{34}\right)^{2}+\left(\frac{6}{34}\right)^{2}+\left(\frac{4}{34}\right)^{2}+\left(\frac{8}{34}\right)^{2}+\left(\frac{12}{34}\right)^{2}\right] \approx 0.048$ for $\mathrm{G}_{1}$ and similarly $Y_{c}=\frac{639}{13005} \approx 0.049$ for $\mathrm{G}_{2}$. Therefore according to our criterion stated in section 3, the performance of the second group was slightly better.

\subsection{Comparison of the Results of the Assessment Methods}

The application of the above - three in total - methods for assessing students' AR skills resulted to different conclusions. In fact, in the first case (calculation of the means) $G_{1}$ demonstrated a better performance than $G_{2}$, while according to the GPA index the two groups demonstrated equal performances. However, according to the TFAM, $\mathrm{G}_{2}$ demonstrated a better performance than $\mathrm{G}_{1}$ !

The above conclusions are not embarrassing at all, since, in contrast to the calculation of the means, which focuses on a group's mean performance, the GPA and the TFAM methods focus on its quality performance by assigning greater coefficients to the higher scores. Further, on comparing the first of equations (2) with equation (4), it turns out that the TFAM assigns greater weight coefficients to the higher scores than the GPA index (see Table 2). In other words, TFAM is more sensitive to the higher scores than the GPA index.

Table 2. Weight coefficients of the $y_{i}$ 's

\begin{tabular}{|c|c|c|}
\hline $\mathrm{y}_{\mathrm{i}}$ & GPA & TRAFM $\left(\mathrm{X}_{\mathrm{c}}\right)$ \\
\hline $\mathrm{y}_{1}$ & 0 & 7 \\
\hline $\mathrm{y}_{2}$ & 1 & 14 \\
\hline $\mathrm{y}_{3}$ & 2 & 21 \\
\hline $\mathrm{y}_{4}$ & 3 & 28 \\
\hline $\mathrm{y}_{5}$ & 4 & 35 \\
\hline
\end{tabular}

In concluding, it is suggested to the user of the above methods to choose the one that it fits better to its personal criteria of goals. However, a combined application of them could help him/her to get a more comprehensive view of the students' performance.

\section{Conclusions and Discussion}

The common experience shows that the assessment methods of a group's performance usually applied in practice are those based on principles of the bivalent logic (yes-no). However, fuzzy logic, due to its nature of characterizing the ambiguous cases with multiple values, offers a wider and richer field of resources for this purpose. This gave us the impulsion to introduce in this paper an extended (with 5 grades) version of the TFAM approach for assessing students' AR skills. TFAM is a recently developed (in a version with 3 grades) variation of the COG defuzzification technique fitting better to the ambiguous cases of students' scores lying at the boundaries between two different student grades.

However, there is a need for more applications to be performed for obtaining safer statistical data. On the other hand, since the TFAM approach looks to have the potential of a general assessment method, our future plans for further research on the subject include also the effort to apply this approach in assessing the individuals' performance in several other human activities.

\section{References}

[1] Gentner, D., Toupin, C., "Systematicity and surface similarity in development of analogy”, Cognitive Science, 10, 277-300, 1986.

[2] Gentner, D., Markman, A. B., "Structure mapping in analogy and similarity”, American Psychologist 52, 45-56, 1997.

[3] Gick, M. L., Holyoak, K. J., “Analogical problem solving”, Cognitive Psychology, 12, 306-355, 1980.

[4] Gick, M. L., Holyoak, K. J., "Schema induction and analogical transfer”, Cognitive Psychology, 15, 1-38, 1983. 
[5] “Grade Point Average Assessment", available in the Web at: http://www.swinburne.edu.au/studentadministration/assessment/gpa.html, visited October 15, 2014.

[6] Holyoak K. J., "The pragmatics of analogical transfer”. In G. H. Bower (Ed.), The psychology of learning and motivation, Vol. 19, 59-87, New York: Academic Press., 1985

[7] Klir, G. J. \& Folger, T. A.., Fuzzy Sets, Uncertainty and Information, Prentice-Hall, London, 1988.

[8] Novick, L. R., "Analogical transfer, problem similarity and expertise”, Journal of Educational Psychology: Learning, Memory and Cognition, 14, 510-520, 1988.

[9] Subbotin, I. Ya., Badkoobehi, H., Bilotckii, N. N., "Application of fuzzy logic to learning assessment", Didactics of Mathematics: Problems and Investigations, 22, 38-41, 2004.

[10] Subbotin, I. Ya., Voskoglou, M. Gr., “Applications of fuzzy logic to Case-Based Reasoning”, International Journal of Applications of Fuzzy Sets and Artificial Intelligence, 1, 7-18, 2011.

[11] Subbotin, I., Mossovar-Rahmani, F., Bilotskii, N., "Fuzzy logic and the concept of the Zone of Proximate Development", Contemporary trends in the development of mathematics and its application aspects (Proceedings of the $1^{\text {st }}$ International Scientific Internet-Conference), pp. 301-302, Donetsk, 2012.

[12] Subbotin, I.Ya., Bilotskii, N. N., "Triangular fuzzy logic model for learning assessment", Didactics of Mathematics: Problems and Investigations, 41, 84-88, 2014.

[13] Subbotin, I. Ya., Voskoglou, M. Gr., "Language, mathematics and critical thinking: The cross influence and cross enrichment", Didactics of Mathematics: Problems and Investigations, 41 89-94, 2014.

[14] Subbotin, I. Ya., Voskoglou M. Gr., "A Triangular Fuzzy Model for Assessing Students' Critical Thinking Skills”, International Journal of Applications of Fuzzy Sets and Artificial Intelligence, 4, 173-186, 2014

[15] Voskoglou, M. Gr., "The process of learning mathematics: A fuzzy set approach”, Heuristic and Didactics of Exact Sciences,, 10, 9-13, 1999

[16] Voskoglou, M. Gr., "Analogical Problem Solving and Transfer”, Proceedings of the $3 d$ Mediterranean Conference on Mathematical Education, 295-303, Athens, 2003.

[17] Voskoglou, M. Gr., "Transition across levels in the process of learning: A fuzzy model”, International Journal of Modelling and Application, Univ. Blumenau, Brazil, 1, 37-44, 2009.

[18] Voskoglou, M. Gr., Stochastic and fuzzy models in Mathematics Education, Artificial Intelligence and Management, Lambert Academic Publishing, Saarbrucken, Germany, 2011.

[19] Voskoglou, M. Gr., "Case-Based Reasoning: History, Methodology and Development Trends", International Journal of Psychology Research, 6 (5), 505-530, Nova Publishers, N. Y., 2011.

[20] Voskoglou, M. Gr, "Fuzzy measures for students' mathematical modelling skills", International Journal of Fuzzy Logic Systems, 2 (2), 13-26, 2012

[21] Voskoglou, M. Gr., Subbotin, I. Ya., "Fuzzy models for analogical reasoning”, International Journal of Applications of Fuzzy Sets and Artificial Intelligence, 2, 19-38, 2012.

[22] Voskoglou, M. Gr., "Assessing the Players' Performance in the Game of Bridge: A fuzzy Logic Approach”, American Journal of Applied Mathematics and Statistics, 2 (3), 115-120, 2014.

[23] Voskoglou, M. Gr., Salem, A.-B. M., "Analogy-Based and CaseBased Reasoning: Two Sides of the Same Coin", International Journal of Applications of Fuzzy Sets and Artificial Intelligence, 4, 5-51, 2014.

[24] Wikipedia, "Center of mass: A system of particles", available in the Web at: http://en.wikipedia.org/wiki/Center_of_mass\#A_system_of_partic les, visited on October 10, 2014.

\section{Appendix}

List of problems of our classroom experiment and their solutions' procedure

CASE 1

Target problem: A box contains 8 balls numbered from 1 to 8 . One makes three successive drawings, putting back the corresponding ball to the box before the next drawing. Find the probability of getting all the balls drawing out of the box different to each other.

Solution's sketch: The probability is equal to the quotient of the total number of the ordered samples of 3 objects from 8 (favourable outcomes) to the total number of the corresponding samples with replacement (possible outcomes).

Remote analogue: How many numbers of 2 digits can be formed by using the digits from 1 to 6 and how many of them have their digits different?

Solution's procedure given to the students: Find the total number of the ordered samples of 2 objects from 6 with and without replacement respectively.

Distracting problem: A box contains 3 white, 4 blue and 6 black balls. If we draw out 2 balls, what is the probability to be of the same colour?

Solution's procedure given to the students: The number of all favourable outcomes is equal to the sum of the total number of combinations of 3, 4 and 6 objects taken 2 at each time respectively, while the number of all possible outcomes is equal to the total number of combinations of 13 objects taken 2 at each time.

Unrelated problem: Find the number of all possible anagrammatisms of the word "SIMPLE". How many of them start with $S$ and how many of them start with $S$ and end with $\mathrm{E}$ ?

Solution's procedure given to the students: The number of all possible anagrammatisms is equal to the total number 6 ! of the permutations of 6 objects. The anagrammatisms starting with $\mathrm{S}$ are 5 ! and the anagrammatisms starting with $\mathrm{S}$ and ending with $\mathrm{E}$ are 4 !

CASE 2

Target problem: Consider the matrices:

$$
\mathrm{A}=\left[\begin{array}{ccc}
1 & -n & -n \\
0 & 1 & -n \\
0 & 0 & 1
\end{array}\right] \kappa \alpha \imath \mathrm{B}=\left[\begin{array}{ccc}
0 & -n & -n \\
0 & 0 & -n \\
0 & 0 & 0
\end{array}\right]
$$

Show that $\mathrm{A}^{\mathrm{n}}=\mathrm{A}+(\mathrm{n}-1)\left(\mathrm{B}+\frac{n}{2} \mathrm{~B}\right)$, for every positive integer $\mathrm{n}$.

Solution's sketch: Since $\mathrm{A}=\mathrm{I}+\mathrm{B}$, where $\mathrm{I}$ stands for the unitary $3 \mathrm{X} 3$ matrix, and $\mathrm{B}^{3}=0$, is $\mathrm{A}^{\mathrm{n}}=(\mathrm{I}+\mathrm{B})^{\mathrm{n}}=\mathrm{I}+\mathrm{nB}+$ $+\frac{n(n-1)}{2} \mathrm{~B}^{2}==\mathrm{A}+(\mathrm{n}-1) \mathrm{B}+\frac{n(n-1)}{2} \mathrm{~B}=\mathrm{A}+(\mathrm{n}-1)\left(\mathrm{B}+\frac{n}{2} \mathrm{~B}\right)$

Remote analogue: Let $\alpha$ be a nonzero real number. Prove that $\alpha^{\mathrm{n}}=\sum_{i=0}^{n}\left(\begin{array}{l}n \\ i\end{array}\right)(a-1)^{n}$, for all positive integers $n$.

Solution's procedure given to the students: Write $\alpha=$ $1+(\alpha-1)$ and apply the Newton's formula $(x+b)^{\mathrm{n}}=$ $\sum_{i=0}^{n}\left(\begin{array}{l}n \\ i\end{array}\right) x^{n-i} b^{i}$, setting $\mathrm{x}=1$ and $\mathrm{b}=\alpha-1$.

Distracting problem: If $\mathrm{A}$ and $\mathrm{B}$ are as in the target problem, calculate $(\mathrm{A}+\mathrm{B})^{2}$.

Solution's procedure given to the students: Make the corresponding calculations.

Unrelated problem: Prove that $1+2+\ldots+n=\frac{n(n+1)}{2}$, for all positive integers $n$.

Solution's procedure given to the students: Apply induction on $n$. 